\title{
Reproductive coercion sometimes works: evaluating whether young African-American women who experience reproductive coercion or birth control sabotage are more likely to become pregnant
}

\author{
Janet E. Rosenbaum ${ }^{1,2}$ D $\cdot$ Ralph J. DiClemente ${ }^{1,2}$
}

Received: 31 March 2020 / Revised: 11 July 2020 / Accepted: 15 July 2020 / Published online: 27 July 2020

(c) Springer Science+Business Media, LLC, part of Springer Nature 2020

\begin{abstract}
Men engaging in reproductive coercion may coerce, force, or deceive female partners into pregnancy. This study evaluates whether the 3-month incidence of pregnancy is higher among women reporting reproductive coercion than similar women reporting no reproductive coercion. We tested this hypothesis in longitudinal data from a sample of African-American women ages 18-24 recruited from community settings in Atlanta, Georgia, US, in 2012-2014 $(n=560)$. Participants were surveyed at baseline, 3 months, 6 months, 9 months, and 12 months. To reduce selection bias, we used full matching on 22 baseline variables related to demographics, economic power, risky alcohol use, and genderbased power inequality. We used logistic regression in the matched sample with outcome pregnancy 3 months later, controlling for baseline fertility intentions $(n=482, n=458$, $\mathrm{n}=452$ at respectively 3,6,9 months). At 3 months, $15 \%$ of women reported reproductive coercion. At 6 months, $11.3 \%$ of women reporting coercion were pregnant vs. $4.6 \%$ of matched women reporting no coercion $(p=0.06)$. Women reporting coercion had 3 times the odds of pregnancy as matched women reporting no coercion (AOR 2.95, 95\% CI (1.16, $6.98), p=0.02$ ). Among women pregnant after coercion, only $15 \%$ wanted to be pregnant then or sooner. Women reporting reproductive coercion are at greater risk of unwanted or mistimed pregnancies, and the semen exposure that caused these pregnancies could also transmit STI/HIV. Clinicians should screen patients for reproductive coercion; consider using semen exposure biomarkers such as PSA or Yc-PCR to identify condom sabotage or stealthing; and refer women experiencing reproductive coercion to supportive services.
\end{abstract}

Keywords Reproductive coercion · Propensity matching · Full matching · Unplanned pregnancy $\cdot$ Intimate partner violence

Electronic supplementary material The online version of this article (https://doi.org/10.1007/s1074 2-020-00213-9) contains supplementary material, which is available to authorized users.

Janet E. Rosenbaum

janet.rosenbaum@downstate.edu

Extended author information available on the last page of the article 


\section{Introduction}

Reproductive coercion is a category of abuse in which male partners interfere with females' reproductive choices, including forced abortions, pregnancy coercion, and contraception sabotage. A recent systematic review estimated that $5-13 \%$ of young adult women attending family planning clinics have experienced reproductive coercion, with greater prevalence among non-Hispanic African-American women (Trister and Anderson 2018). Women and adolescents may experience reproductive coercion as part of violent or abusive relationships (Pallitto et al. 2005; Miller et al. 2010; Paterno and Jordan 2012; Silverman et al. 2011; Hathaway et al. 2005), but reproductive coercion may also occur even in the absence of other relationship abuse (ACOG 2013). Even studies that have not explicitly measured reproductive coercion have found that young African-American women who rely on a boyfriend for spending money source or for housing are less likely to have safe sex, suggesting that these implicit forms of reproductive coercion may potentially undermine otherwise effective HIV prevention interventions (Rosenbaum et al. 2012, 2016b). Whether implicit or overt, reproductive coercion or birth control sabotage markedly undermines the capacity of safe sex interventions to promote safer sex, reduce unplanned pregnancy, and reduce HIV/STI.

This study estimates the 3-month and lifetime incidence of reproductive coercion and birth control sabotage using longitudinal data in a sample of women at high risk of HIV and evaluates whether women's experience of reproductive coercion predicts subsequent pregnancy.

\section{Methods}

This study is an observational study using data from a randomized experiment to evaluate an HIV prevention intervention for alcohol-using African-American women ages 18-24. The present study does not evaluate the intervention itself (Clinical trial registration number: NCT01553682.)

\subsection{Data}

Trained African-American community outreach workers recruited African-American women from January 2012 to February 2014 in Atlanta, Georgia. Baseline data was collected between March 3, 2012 and February 8, 2014, with the final (12-month) follow-up assessment data was collection between March 9, 2013 and February 13, 2015, after which the study ended as planned. Recruitment staff approached potentially eligible young women for confidential screening or scheduled them for later phone screening. A female AfricanAmerican recruitment coordinator described the study, solicited participation, and assessed women's eligibility and interest in participating in the project. Young women were eligible to participate in the study if they self-identified as African-American, were 18-24 years old, not married, tested negative for pregnancy with a urine pregnancy test, had consumed alcohol on at least three occasions in the past 90 days, had unprotected vaginal or anal sex with a male in the past 90 days, and did not present with active psychosis or a severe illness that could preclude participation in the program and follow-up assessments. Of the eligible women, $96 \%(\mathrm{~N}=560)$ enrolled in the study, completed baseline assessments, and were 
randomized to study conditions in the three-arm trial. The two treatment arms were HIVprevention interventions; the control arm was an equivalent-hour nutritional intervention.

The participants' median age was 20 (interquartile range 19-22); two-thirds had a high school diploma or more; $27 \%$ were employed; and $83 \%$ received Supplemental Nutrition Assistance Program benefits (food stamps) (Table 1). Among participants, $85 \%$ had a current boyfriend, $51 \%$ were monogamous in the past 3 months, $21 \%$ perceived that their partner had another partner in the past 3 months, $21 \%$ tested positive for chlamydia or gonorrhea, and $16 \%$ were trying to get pregnant.

Respondents were surveyed at baseline and every 3 months afterwards through the 12 months post-baseline assessment, a total of 5 waves. As a percentage of the baseline sample $(\mathrm{n}=560)$, the participation percentages were $86 \%$ at 3 months, $82 \%$ at 6 months, $81 \%$ at 9 months, and $83 \%$ at 12 months; $67 \%$ participated in all 4 follow-up waves. At each wave, participants completed an interview administered via audio computer-assisted self-interviewing (ACASI) and were tested for chlamydia, gonorrhoea, and trichomonas on a self-administered vaginal swab that was assayed using polymerase chain reaction procedures. At baseline, women provided a urine sample to assess pregnancy. The ACASI required participants to answer all questions that they were asked, so the only missing data were due to wave non-response or investigator-programmed skip patterns, including errors. At the 6-month follow-up assessment, pregnancy status was missing for 7 participants due to a programming error and so missing completely at random; all other variables at other follow-up assessments were present for all participants.

Women who reported coercion/sabotage or pregnancy did not differ in the likelihood of participating in subsequent survey waves (not shown). However, non-participating women who were currently experiencing coercion/sabotage or pregnancy may have been less likely to participate in that follow-up assessment, so we could not use data analysis techniques that assume that data are missing at random, such as multiple imputation or generalised estimating equations. The analysis of reproductive coercion at 3 months included 482 women, of whom 71 reported coercion/sabotage; at 6 months, the analysis included 458 women, of whom 38 reported coercion/sabotage; and at 9 months, the analysis included 452 women, of whom 30 reported coercion/sabotage.

Participants received an incentive of $\$ 60$ for each 4-hour session, $\$ 50$ for each follow-up survey, and $\$ 5$ for each intervention-related telephone session or text message. Emory University's Institutional Review Board approved all study protocols (IRB00048502).

\subsection{Measures}

\subsubsection{Temporal ordering of variables}

To make the temporal ordering of events clear, the control variables were measured at baseline. The predictor was a binary indicator for coercion/sabotage measured at 3 months, and the outcome of pregnancy was measured at 6 months. Likewise, for coercion/sabotage measured at 6 months, pregnancy was measured at 9 months; for coercion/sabotage measured at 9 months, pregnancy was measured at 12 months.

\subsubsection{Control variables}

We used Connell's Theory of Gender and Power (Connell 1987) to identify baseline variables that were potential confounders of the association between coercion/sabotage and 
Table 1 Demographic composition of sample $(n=560)$

\begin{tabular}{|c|c|}
\hline & No. $(\%)$ \\
\hline Age in years (mean (SD)) & $20.6(1.90)$ \\
\hline Age 17 & $1(0.2)$ \\
\hline Age 18 & $101(18.0)$ \\
\hline Age 19 & $88(15.7)$ \\
\hline Age 20 & $96(17.1)$ \\
\hline Age 21 & $83(14.8)$ \\
\hline Age 22 & $79(14.1)$ \\
\hline Age 23 & $72(12.9)$ \\
\hline Age 24 & $40(7.1)$ \\
\hline \multicolumn{2}{|l|}{ Highest educational attainment } \\
\hline Less than high school diploma/GED & $185(33.0)$ \\
\hline Graduated high school or GED & $231(41.3)$ \\
\hline Some college & $128(22.9)$ \\
\hline Graduated college & $11(2.0)$ \\
\hline Other & $5(0.9)$ \\
\hline Employed & $152(27.1)$ \\
\hline \multicolumn{2}{|l|}{ Public assistance } \\
\hline Welfare (e.g., temporary assistance to needy families) & $80(14.3)$ \\
\hline Supplemental Nutrition Assistance Program (food stamps) & $466(83.2)$ \\
\hline WIC & $153(27.3)$ \\
\hline Section 8 & $59(10.5)$ \\
\hline \multicolumn{2}{|l|}{ Relationship characteristics } \\
\hline Current boyfriend & $473(84.5)$ \\
\hline Monogamous in past 3 months & $287(51.3)$ \\
\hline Perceived partner concurrency in past 3 months (main partner had another partner) & $116(20.7)$ \\
\hline Trying to get pregnant & $89(15.9)$ \\
\hline \multicolumn{2}{|l|}{ Relative age of sex partners } \\
\hline About the same age or younger & $233(41.6)$ \\
\hline 2-3 years older & $202(36.1)$ \\
\hline More than 4 years older & $125(22.3)$ \\
\hline \multicolumn{2}{|l|}{ History of abuse (ever) } \\
\hline Emotional & $241(43.0)$ \\
\hline Physical & $178(31.8)$ \\
\hline Use marijuana daily & $95(17.0)$ \\
\hline Tested positive for chlamydia or gonorrhea & $117(20.9)$ \\
\hline Ever douche & $291(52.0)$ \\
\hline Assigned to HIV-prevention intervention & $375(67.0)$ \\
\hline Participated in all 4 follow-up surveys & $373(66.6)$ \\
\hline \multicolumn{2}{|l|}{ Number of surveys reported coercion/sabotage in past 3 months+ } \\
\hline 0 surveys & $177(47.5)$ \\
\hline 1 survey & $128(34.3)$ \\
\hline 2 surveys & $47(12.6)$ \\
\hline 3 surveys & $14(3.8)$ \\
\hline 4 surveys & $5(1.3)$ \\
\hline 5 surveys & $2(0.5)$ \\
\hline
\end{tabular}


Table 1 (continued)

The variables in the categories public assistance, relationship characteristics, and history of abuse are not mutually exclusive

+ Number of surveys with reported coercion/sabotage in past 3 months is among the 373 participants who participated in all 4 follow-up surveys

pregnancy. Potential confounding variables are associated with both coercion/sabotage and with pregnancy and are not intermediate variables between coercion/sabotage and pregnancy. In his Theory of Gender and Power, Connell defined three structures as fundamental to relationships between men and women - sexual division of labor, sexual division of power, and cathexis (emotional attachment within relationships, social norms) — and noted that these three structures apply at the societal and institutional levels (Connell 1987). Wingood and DiClemente adapted this theory to HIV prevention and identified HIV risks within each structure (2000). The sexual division of labor includes economic exposures such as poverty and socioeconomic exposures such as minority status or youth: related control variables include age, neighborhood quality, educational attainment, and employment status. The sexual division of power includes physical exposures including relationship abuse and substance abuse history: related control variables include having a history of sexual abuse, physical abuse, exchange sex, marijuana use, AUDIT score assessing problematic alcohol use, and usual level of alcohol use. Cathexis comprises "social norms and affective attachments" and includes social exposures and psychological distress: related control variables were children, pregnancy history, fertility intentions, age difference with boyfriend, and boyfriend having concurrent partners (Wingood and DiClemente 2000).

\subsubsection{Predictor}

Reproductive coercion and birth control sabotage were assessed both for lifetime incidence ("ever") and in the past 3 months. Participants were only asked each coercion/sabotage item in the past 3 months if they endorsed the corresponding item asking about lifetime coercion/sabotage. Reproductive coercion was assessed as endorsing any of 4 items, "someone you were dating or going out with ...": (1) "told you not to use any birth control (like the pill, shot, ring, etc.)"; (2) "said he would leave you if you did not get pregnant"; (3) "told you he would have a baby with someone else if you didn't get pregnant"; (4) "hurt you physically because you did not agree to get pregnant" (Miller et al. 2010). Birth control sabotage was assessed as endorsing any of 5 items: (1) "taken off the condom while you were having sex so that you would get pregnant"; (2) "put holes in the condom so you would get pregnant"; (3) "broken a condom on purpose while you were having sex so you would get pregnant"; (4) "taken your birth control (like pills) away from you or kept you from going to the clinic to get birth control so that you would get pregnant"; (5) "made you have sex without a condom so you would get pregnant." (Miller et al. 2010) For the matched sampling analysis, we combined the two measures into a single binary measure because they load onto a single factor in confirmatory factor analysis. For the combined 9 items, internal consistency as assessed by Cronbach's alpha was 0.75, 0.82, 0.76, and 0.75 at baseline, 3, 6, and 9 months, which is considered moderate-high internal consistency and as high or higher as the internal consistencies reported in the systematic review of reproductive coercion (Kolenikov and Angeles 2004; Trister and Anderson 2018). 


\subsubsection{Outcomes}

This study evaluates pregnancy at the 6, 9, and 12 month follow-up assessments from self-report response to the question "Are you currently pregnant?" We used pregnancy status at 3 months only for bivariate analysis because there were no pre-coercion/sabotage variables available; pregnancy at 3 months was missing for 72 individuals due to an error in the survey's skip patterns.

To understand the context of reported pregnancies, we assessed respondents' fertility intentions just prior to pregnancy with the question "Thinking back to just before you got pregnant this last time, how did you feel about becoming pregnant?" with possible responses: "I wanted to be pregnant sooner"; "I wanted to be pregnant then"; "I wanted to be pregnant later"; and "I didn't want to be pregnant then or at any time in the future." Prenatal care was assessed as a yes/no question "Have you received prenatal care during this pregnancy?" Gestational length of pregnancy in weeks was calculated in Stata using the self-reported due date and the assessment date, based on an average gestational length of 280 days.

\subsection{Data analysis}

\subsubsection{Matching}

We identified a comparison group of women who did not experience reproductive coercion or birth control sabotage that are similar to women who did using full matching as implemented by the R MatchIt package (Ho et al. 2018). Full matching estimates propensity scores as the fitted values from a logistic regression with the outcome having experienced reproductive coercion or birth control sabotage and creates groups of observations with similar estimated propensity scores. Observations in the non-coercion group with estimated propensities of coercion/sabotage that were outside the range of estimated propensity score for the coercion/sabotage group were omitted. In this case, full matching preserved the full sample for all three pairs of coercion-pregnancy measures. For coercion/sabotage at 3 months and pregnancy at 6 months, 61 women reporting coercion/sabotage were matched to 365 women reporting no coercion/sabotage. For coercion at 6 months, 35 women reporting coercion/sabotage were matched to 391 women reporting no coercion/sabotage. For coercion at 9 months, 30 women reporting coercion/sabotage were matched to 422 women reporting no coercion/sabotage.

\subsubsection{Analysis after matching}

We assessed balance by computing the standardised mean differences for each control variable. The standardised mean differences are the difference in means divided by the pooled standard deviation, a measure that is not sensitive to sample size. The coerced and non-coerced groups were determined to be balanced if the standardised mean differences were less than 0.2 , and ideally less than 0.1 .

We followed Rubin's recommendation to estimate treatment effects only once, after determining that the best possible balance had been achieved with full matching. In the matched sample, we tested for differences in pregnancy prevalence between groups 
using Fisher's exact test and predicted pregnancy in the matched sample using logistic regression, controlling for baseline fertility intentions.

We used causal mediation analysis with the $\mathrm{R}$ mediation package (Imai et al. 2010; Tingley et al. 2014) to evaluate whether baseline fertility intentions explained the association between reproductive coercion/sabotage and pregnancy.

We used sensitivity analysis for full matching in the R sensitivityfull package to quantify unobserved confounding between coercion/sabotage at 3 months and pregnancy at 6 months (Rosenbaum 2007). The sensitivity analysis uses a randomization test to evaluate whether there appears to be departures from random assignment at each level of gamma $(\Gamma)$. The parameter $\Gamma$ is a bound on the odds ratios measuring the associations between the unobserved variable and coercion/sabotage, and between the unobserved variable and pregnancy that would make the association between coercion/sabotage and pregnancy no longer significant (Liu et al. 2013). That is, the sensitivity analysis assesses the likelihood that the association that we observe between coercion/sabotage and subsequent pregnancy could be due an unobserved confounding variable. A larger $\Gamma$ implies lower potential for confounding by an unobserved confounding variable. Due to few individuals reporting coercion/sabotage at 6 and 9 months, possibly due to under-report, we were unable to use this sensitivity analysis procedure for the analyses with predictor coercion/sabotage at 6 months and 9 months, with outcomes pregnancy at respectively 9 months and 12 months.

Due to heterogeneity in reporting coercion/sabotage by follow-up assessment, we analysed the data separately for each pair of time points and plotted the results using Gelman and Hill's "secret weapon" to plot a key exposure variable's regression coefficient with 95\% confidence interval for separate regressions (Gelman and Hill 2007.) In this case, we plotted the coefficient for the coercion/sabotage variable in logistic regressions predicting pregnancy 3 months later.

The data analysis used Stata SE 15.1 and R 3.5.3 using the gplots library (Warner et al. 2019).

\section{Results}

At baseline, 23\% reported having ever experienced any reproductive coercion; $39.5 \%$ reported having ever experienced birth control sabotage; and $45.9 \%$ reported having ever experienced either coercion or sabotage. Incidence of reproductive coercion or birth control sabotage in the past 3 months were $46 \%, 15 \%$, and $8 \%$, and $7 \%$ at the baseline, 3-, 6-, and 9- month waves, and was not affected by the intervention (Fig. 1). Among participants in all surveys, 53\% reported either reproductive coercion or birth control sabotage in the past 3 months at least once (Table 1).

Among women reporting coercion or sabotage in the past 3 months, 13\%, 18\%, and $19 \%$ were pregnant 3 months later, versus $5 \%, 5 \%$, and $6 \%$ among women reporting no reproductive coercion $(p<0.05)$ (Fig. 2). Among those pregnant at 6, 9, and 12 months, the percentages reporting coercion or sabotage 3 months earlier were $29.6 \%, 25.0 \%$, and $16.7 \%(p<0.05)$ (Table 2). Fertility intentions just prior to pregnancy were not associated with coercion/sabotage. Among pregnant respondents, $15 \%, 17 \%$, and $27 \%$ respectively reported that they wanted to be pregnant then or sooner. Receipt of prenatal care was not associated with reproductive coercion/sabotage. Among pregnant respondents, $81 \%, 67 \%$, and $83 \%$ respectively reported receiving prenatal care. Pregnant respondents were respectively 22.5 weeks (IQR 18-26 weeks), 25 weeks (IQR 12-36), and 24 weeks (IQR 16-31) 


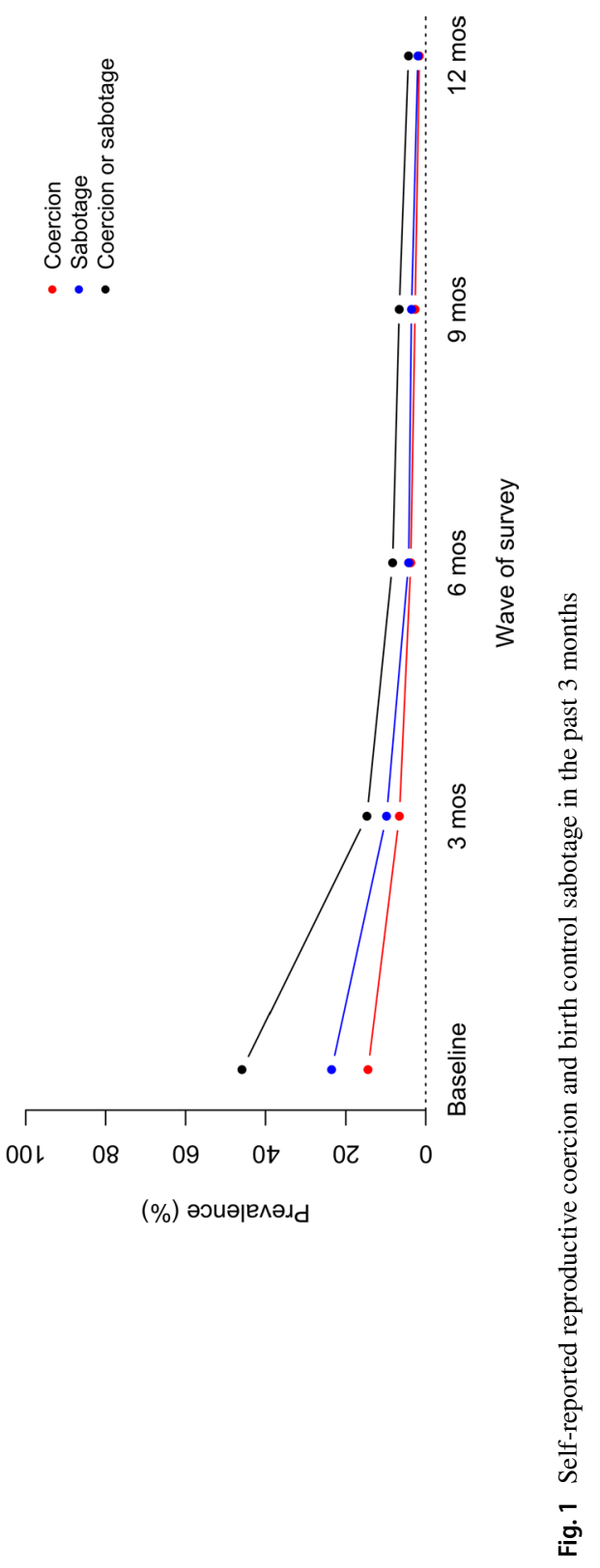




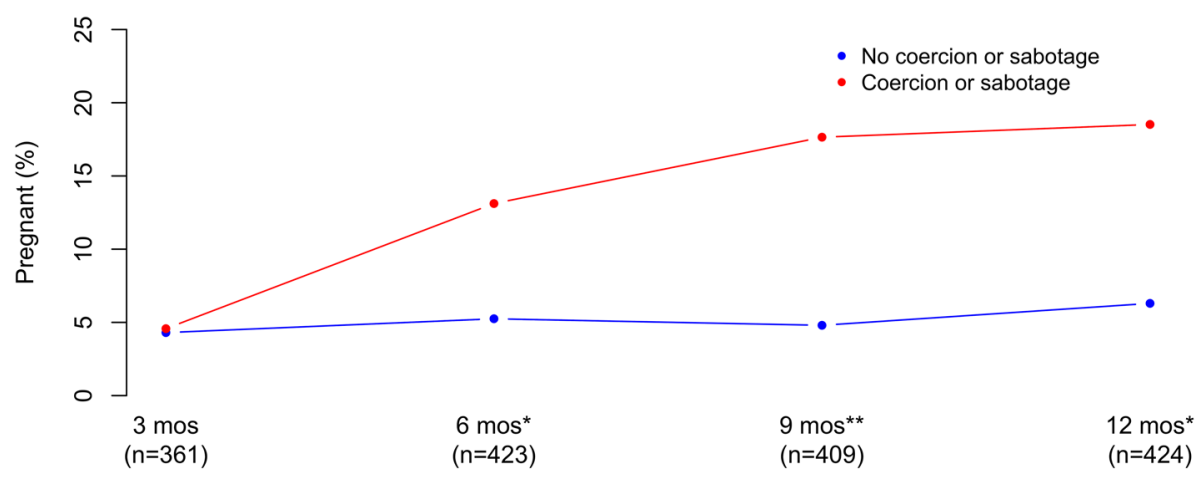

Fig. 2 Pregnancy by reproductive coercion or birth control sabotage in the past 3 months before matching in bivariate analysis. $*=p<0.05 ; * *=p<0.01$

Table 2 Associations between reproductive coercion or birth control sabotage and pregnancy 3 months later

\begin{tabular}{llllll}
\hline & & \multicolumn{2}{l}{ Pregnant 3 months later, No. (column \%) } & P \\
\cline { 3 - 5 } & & Not pregnant & Pregnant & Total \\
\hline Coercion/sabotage at 3 months & None & $346(86.7)$ & $19(70.4)$ & $365(85.7)$ & 0.02 \\
& Any & $53(13.3)$ & $8(29.6)$ & $61(14.3)$ & 0.002 \\
Coercion/sabotage at 6 months & None & $357(92.7)$ & $18(75.0)$ & $375(91.7)$ & $34(8.3)$ \\
& Any & $28(7.3)$ & $6(25.0)$ & $398(93.7)$ & 0.02 \\
Coercion/sabotage at 9 months & None & $373(94.4)$ & $25(83.3)$ & $27(6.4)$ & \\
& Any & $22(5.6)$ & $5(16.7)$ & $27)$ & \\
\hline
\end{tabular}

The $p$ value is from a Chi square test with 1 degree of freedom, evaluating the association between reproductive coercion with pregnancy 3 months later

pregnant. For all waves, coercion/sabotage in the past 3 months and pregnancy were both not associated with non-participation in the following survey (not shown).

Baseline factors associated with greater risk of reproductive coercion at 3 months included baseline coercion/sabotage, risky alcohol use, lower economic power, exchange sex, having an older partner, and history of emotional and physical abuse; protective factors included monogamy, being a high school graduate, and usually drinking 2 or fewer drinks. Full matching methods resulted in balance on all covariates plus propensity score and preserved the full sample size for coercion/sabotage at 3 and 6 months; balance was not achieved at 9 months, but the direction of bias for the one unbalanced variable was reversed by matching (Figs. 3, 4, 5).

After matching, among women reporting coercion/sabotage at each of the three time periods, pregnancy was more common in the group reporting coercion/sabotage: at 6 months, $11.3 \%$ were pregnant among women who had reported coercion/sabotage at 3 months versus $4.6 \%$ were pregnant among the matched group reporting no coercion/sabotage (Fisher's exact test $p=0.06$ ); women reporting coercion/sabotage had nearly 3 times the odds of pregnancy as the matched non-coerced/sabotaged women (OR 2.95, 95\% CI $(1.16,6.98), p=0.02$ ) (Fig. 6). This association is not mediated by baseline fertility intentions, according to causal mediation analysis. 
Propensity score

Coercion/sabotage past $3 \mathrm{~m}$

AUDIT score

Ever emotional abuse

Ever physical abuse

Exchange sex

Partner 4+ years older

Number lifetime partners

Trying to conceive

Ever douche

Boyfriend has other partners

Use marijuana daily

Have a boyfriend

Any children

Ever pregnant

Chlamydia or gonorrhea

Age

Partner 2-3 years older

Treatment

Employed

Drink 2 or fewer drinks

High school graduate

Monogamy, past $3 \mathrm{~m}$

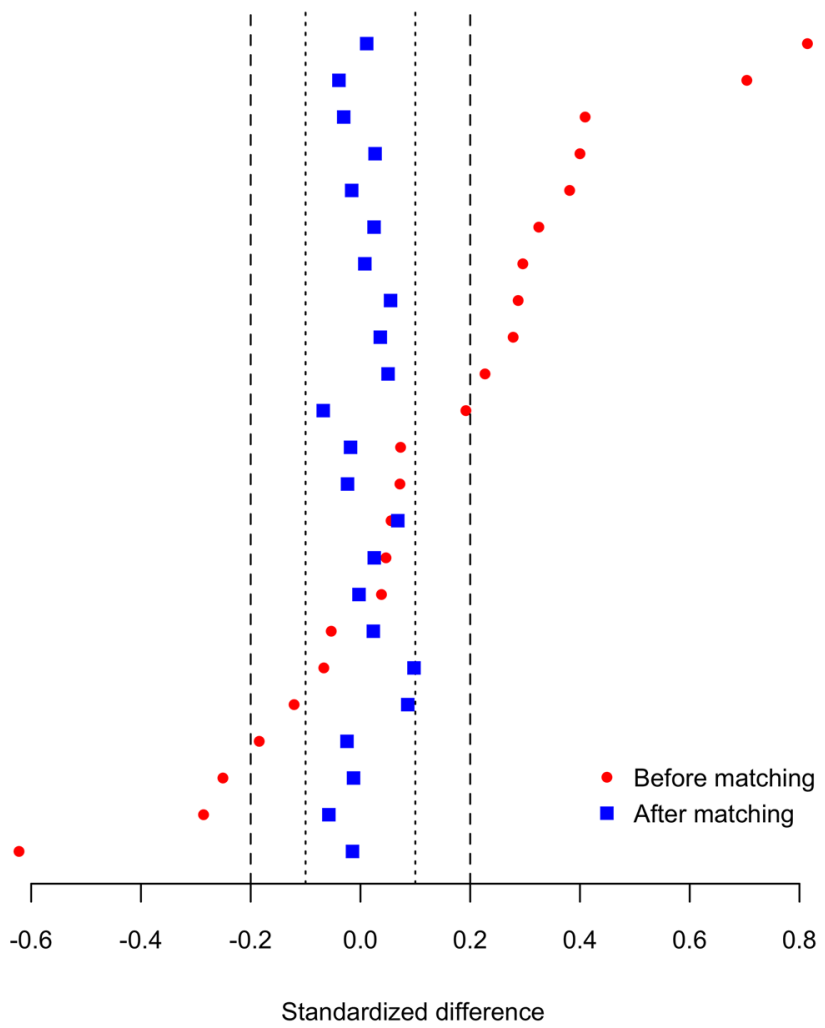

Fig. 3 Standardized differences for baseline variables between women reporting any reproductive coercion or birth control sabotage at 3 months, compared with women reporting no reproductive coercion or birth control sabotage, before and after full matching

The difference in pregnancy in the subclasses formed by full matching on coercion/sabotage status at 3 months was statistically significant under the assumption that matching addressed all important covariates $(p=0.02)$, and it became insignificant under $\Gamma=1.2$, suggesting that this association between coercion/sabotage and pregnancy is insensitive to unobserved biases that increase the chances of coercion/ sabotage by up to $20 \%$ but not larger unobserved biases. That is, if the unobserved variables produce only small impacts on a woman's chance of experiencing reproductive coercion or birth control sabotage, the association between coercion/sabotage and pregnancy would remain.

At 9 months, $15.8 \%$ were pregnant among women who had reported coercion/sabotage at 6 months versus $4.3 \%$ among the matched group $(p=0.02)$; women reporting coercion/sabotage had nearly 4 times the odds of pregnancy as the matched noncoerced/sabotaged women (OR 3.80, 95\% CI (1.30, 9.85), $p=0.009)$. At 12 months, $16.7 \%$ were pregnant among women who reported coercion/sabotage vs. $5.9 \%$ among the matched group ( $p=0.03$ ); women reporting coercion/sabotage had 6 times the odds of pregnancy as the matched non-coerced/sabotaged women (OR 6.44, 95\% CI (1.93, $18.8), p=0.001)$. 


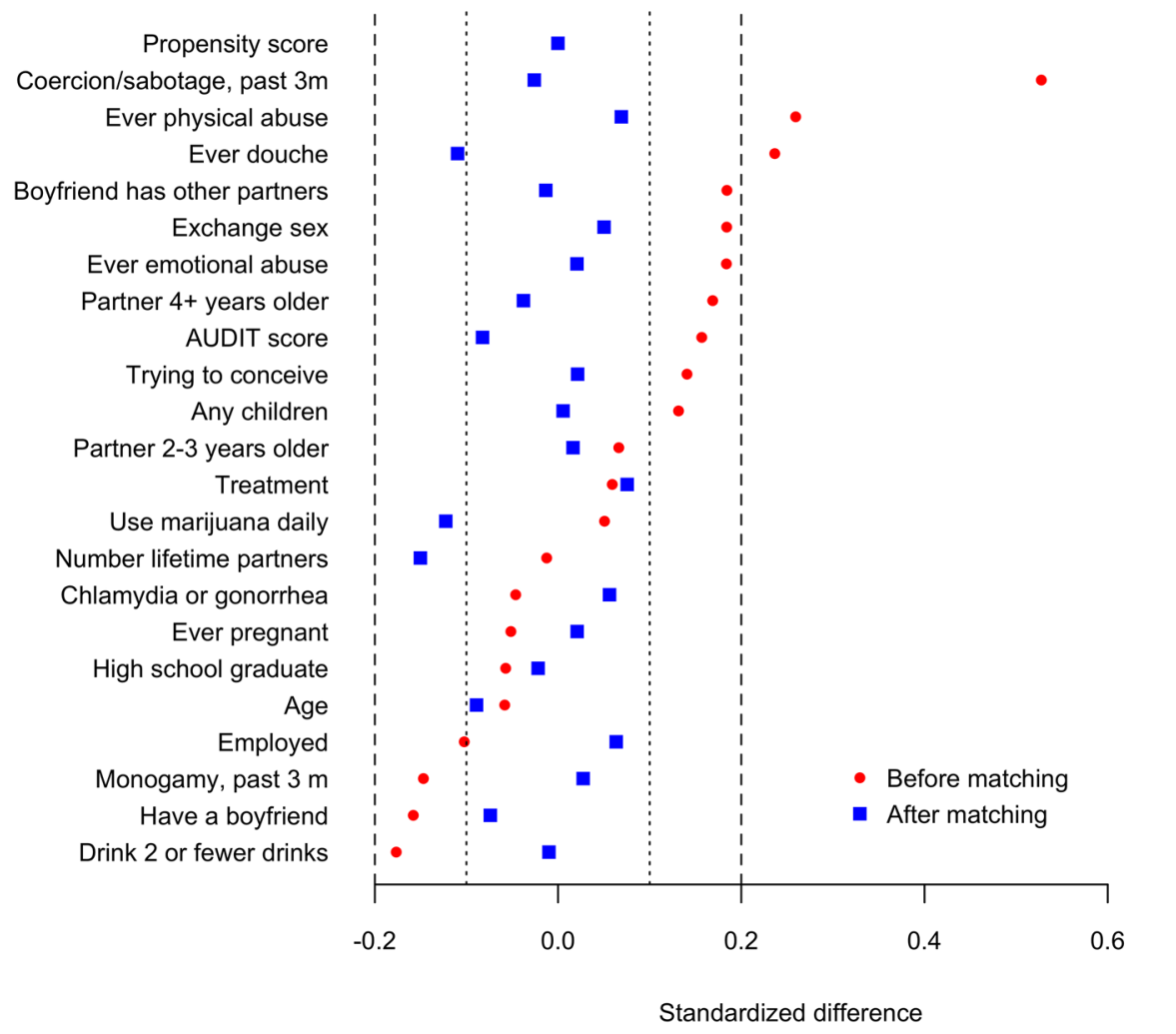

Fig. 4 Standardized differences for baseline variables between women reporting any reproductive coercion or birth control sabotage at 6 months, compared with women reporting no reproductive coercion or birth control sabotage, before and after full matching

\section{Discussion}

\subsection{Findings and Interpretation}

Reproductive coercion and birth control sabotage were more common among these young women recruited in community and street settings for an HIV/STI intervention than in past studies recruited from family planning clinics (Trister and Anderson 2018). Coercion/sabotage predicts greater risk of pregnancy, indicative of unprotected sex that jeopardises the effectiveness of HIV prevention interventions. Between 1/6 and 1/4 of observed pregnancies were among women who reported coercion/sabotage 3 months earlier. These pregnancies appear to be unplanned: the association persisted after matching on baseline fertility intentions and they were not mediated by baseline fertility intentions; also, few pregnant women reported that they wanted to be pregnant then or sooner. Comparing young women with recent coercion/sabotage with other young women without coercion/sabotage, but with similar relationship power inequalities, abuse histories, and other factors, women who experienced coercion or sabotage were more likely to become pregnant; that is, the association is not explained by baseline 
Propensity score

Coercion/Sabotage, past $3 \mathrm{~m}$ AUDIT score

Ever douche

Ever physical abuse

Boyfriend has other partners

Have a boyfriend

Chlamydia or gonorrhea

Partner 2-3 years older

Any children

Ever pregnant

Partner 4+ years older

Ever emotional abuse

Treatment

Number lifetime partners

Employed

Use marijuana daily

Monogamy, past $3 \mathrm{~m}$

High school graduate

Exchange sex

Age

Trying to conceive

Drink 2 or fewer drinks

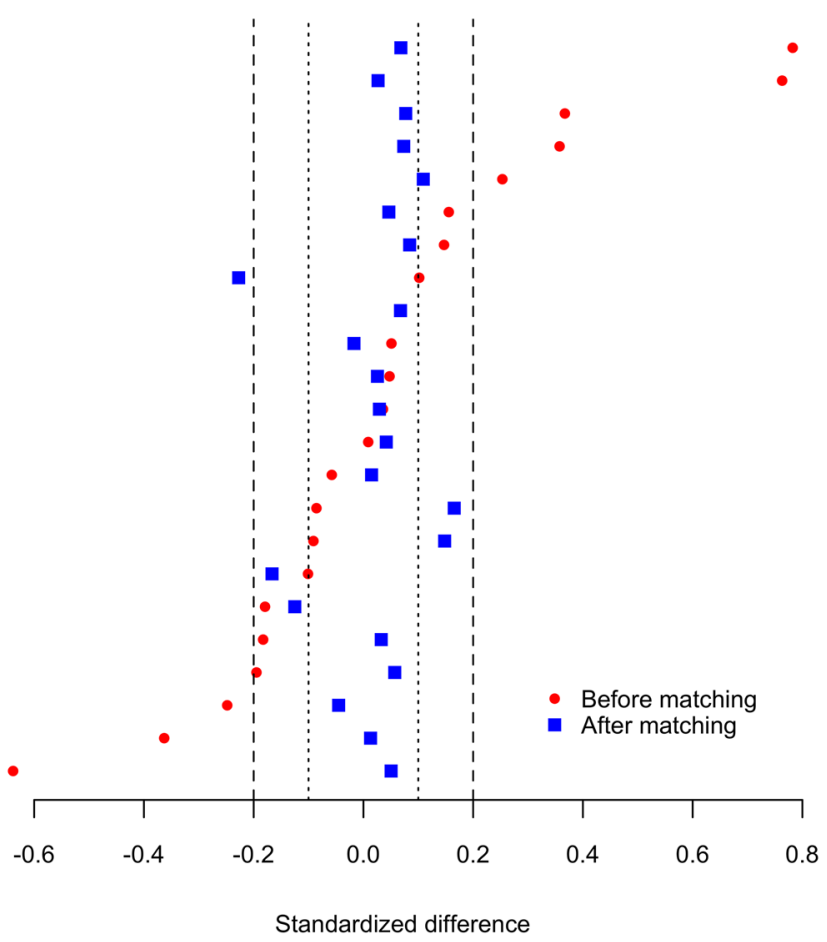

Fig. 5 Standardized differences for baseline variables between women reporting any reproductive coercion or birth control sabotage at 9 months, compared with women reporting no reproductive coercion or birth control sabotage, before and after full matching

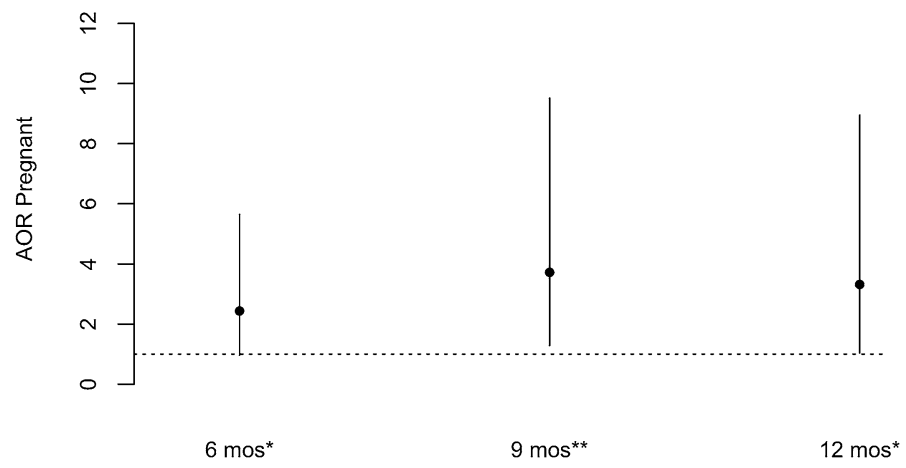

Fig. 6 Association between reproductive coercion or birth control sabotage and pregnancy 3 months later in matched sample, adjusted for baseline fertility intentions. $*=p<0.05 ; * *=p<0.01$

gender-power variables. The sensitivity analysis found that the association is robust to small biases due to unobserved variables, but it is sensitive to moderate biases.

Our past research suggests that reproductive coercion has an economic dimension: we found Black female adolescents and emerging adults who rely on their partners for spending money, transportation, or housing are more likely to never use condoms and 
have unplanned pregnancies (Rosenbaum et al. 2012, 2016b), but that employment is associated with reduced reliance on partners for spending money (Rosenbaum et al. $2014 a, b)$. We expect that the economic recession induced by the COVID-19 pandemic will worsen reproductive coercion and birth control sabotage due to limited options: after decades of wage declines for Black women, the employment and earnings of Black women were disproportionately reduced during the previous economic recession (Laird 2017; Pettit and Ewart Pettit and Ewert 2009).

The U.S.'s increasing restrictions on women's access to reproductive health care, including abortion, compound the lack of agency for women who became pregnant due to coercion or deception. These women live in Georgia, which has a 24-hour waiting period prior to abortion and no public funding for abortion in general circumstances; in 2019, Georgia banned abortion after the detection of a fetal pole heartbeat (Guttmacher 2020).

Coercion and sabotage undermine the goals of preventing pregnancy and HIV/STI, and this CDC-endorsed evidence-based intervention did not reduce coercion or sabotage. Combining HIV-prevention interventions with interventions that reduce reproductive coercion (Miller et al. 2011) may improve HIV intervention effectiveness, and would also target women with the highest prevalence of coercion and sabotage. However, coercion/sabotage is likely to be difficult to reduce because it results from many structural factors, including African-American men's exogamy and African-American women's preference for endogamy (Crowder and Tolnay 2000) and high and increasing cost of living in Atlanta and other urban areas (Rosenbaum et al. 2016a). Coercion/sabotage also results from numerous structural factors related to systemic racism, including employment discrimination producing low earnings for Black women and the school-to-prison pipeline and mass incarceration of Black men (Pettit and Ewart 2009; Rosenbaum 2020). Mass incarceration has produced low male/female gender ratios among young adults, which has been proposed as an explanation for the disproportionately high rates of HIV/STIs among non-Hispanic African-Americans (Pouget et al. 2010).

Self-presentation bias may inhibit some women from reporting lifetime history of coercion and sabotage, suggesting that coercion and sabotage may be under-reported at all waves. The intervention did not decrease coercion/sabotage. Answering the survey may have altered women's behaviour: repeatedly answering questions about coercion/sabotage could have spurred women to end or otherwise alter relationships with partners who engage in coercion/sabotage. It seems unlikely that coercion or sabotage was over-reported at baseline, or that over-reporting would decrease so drastically because sexual victimization is not generally over-reported and the survey conditions were identical across waves (Tourangeau and McNeeley 2003).

Women may not be aware of birth control sabotage that partners may have hidden from them successfully: "stealthing" behaviours such as deceiving women about whether a condom is used, removing condoms, putting holes in condoms, or hiding contraception may occur without women's knowledge. Stealthing has been documented when male partners remove condoms or deceive their male (Klein 2014) or female (Brodsky 2017; Rowlands and Walker 2019) partners about condom use. Qualitative evidence suggests that women may not notice that there is no condom or that the condom was removed, but that some have learned to check for the continued presence of a condom during sex (Teitelman et al. 2011). Because stealthing often occurs without women's knowledge, self-report will underestimate semen exposure, and biomarkers of semen exposure are crucial for interventions that intend to decrease both STI/HIV and unplanned pregnancy (Gallo et al. 2013; Snead et al. 2014). 
Lifetime incidence and 3-month incidence of coercion/sabotage are highly correlated, which is consistent with past research finding that respondents answer survey questions about their past by referencing their current behaviour rather than their actual past behaviour (Collins et al. 1985; Rosenbaum 2006). Surveys do not appear to gain any useful information by asking about the lifetime incidence of coercion or sabotage after the baseline wave; after baseline, surveys should ask only about coercion/sabotage in the past 3 months.

\subsection{Strengths and limitations}

This study is the first to our knowledge to use longitudinal data and to demonstrate an association between reproductive coercion/sabotage and pregnancy using robust statistical methods such as matched sampling to reduce confounding on observed variables and to quantify the sensitivity of results to unobserved variables.

This sample may be more representative of African-American young women in urban areas because these women were recruited from street and community settings, rather than family planning clinics. Women at family planning clinics are self-selected for having been able to attend the clinic, irrespective of their partner's attempts to keep them from seeking contraception or other sexual and reproductive health care. Women at family planning clinics likely have greater average health-related self-efficacy, knowledge, and engage in more health-protective behaviours.

The decrease in recent coercion/sabotage may represent an actual decline in recent coercion/sabotage. However, the decline in coercion/sabotage may be due to increased reluctance to report coercion/sabotage. We did not find an association between baseline coercion/sabotage and lower participation in follow-up waves; however, women experiencing new (i.e., unmeasured) coercion/sabotage may have reduced likelihood of participation in follow-up waves of the study. If self-presentation bias inhibits reporting of coercion/ sabotage, that implies substantial measurement error (Rosenbaum 2009), adding noise that biases results towards the null (Copeland et al. 1977; Haine et al. 2018; Höfler 2005). In spite of potentially high measurement error, this study observed an association between coercion/sabotage and subsequent pregnancy for all 3 assessed follow-up time periods, after matching on baseline covariates, including baseline coercion, sabotage, and fertility intentions.

This study used matched sampling to minimise confounding on baseline relationship inequalities, following best practices of using theory to guide the choice of matching variables and reporting balance (Austin 2008; Ali et al. 2015). Confounding may remain on unobserved or unmatched variables; however, we used sensitivity analysis to quantify potential confounding due to unmeasured variables, and we found that the association between coercion/sabotage and pregnancy was robust to small biases but not large biases.

Finally, the survey was designed to assess health risk behaviors, such as alcohol misuse and safe sex behaviors, but the data omit important information about employment and schooling that may moderate the association between coercion/sabotage and unplanned pregnancy (Rosenbaum et al. 2014a, b). Women who have greater potential earnings, even if they are not currently employed, may be more likely to avoid pregnancy or discontinue coercive romantic relationships. However, we do not know important indicators of participants' future educational attainment and earnings, including high school grades, educational expectancies, or whether these participants are enrolled in college. The survey's measure of educational attainment omitted post-secondary certificates and associates degree categories, which yield greater economic and non-economic returns than a 
high school diploma alone (Belfield and Bailey 2011; Rosenbaum 2012; Rosenbaum and Rosenbaum 2016), and could thus expand women's options to avoid coercive relationships (Rosenbaum et al. 2014a, b).

\subsection{Similarities and differences in relation to other studies}

Nearly half of women reported reproductive coercion or birth control sabotage in the past 3 months in this sample, a much higher percentage than the 5-11\% 3-month incidence in past research in large diverse samples recruited from family planning clinics (McCauley et al. 2017; Miller et al. 2011, 2016). Women who have experienced coercion/sabotage may be under-represented in family planning clinic samples because of the coercion/sabotage.

\subsection{Implications for practice and research}

In a policy environment of increasing legal restrictions on women's autonomy to end unwanted and mistimed pregnancies, clinicians should screen patients for reproductive coercion and birth control sabotage and refer patience to supportive services. To augment self-report, clinical settings could consider using prostate-specific antigen (PSA) to identify potential birth control sabotage in the past $24-48 \mathrm{~h}$ because the PSA is already widely available in clinical settings and costs about $\$ 5$ per test (Gallo et al. 2013). Testing for Y-chromosome DNA using Yc-PCR yields a 7-14 day detection window for semen exposure (Zenilman et al. 2005), but it costs about $\$ 20$ per test and is not widely available in clinical settings (Gallo et al. 2013). Past research has found that young women who were recruited from family planning clinics who test positive for Yc-PCR are more likely to have unplanned pregnancies (Rosenbaum et al. 2014). This study adds to that research and suggests that developing a Yc-PCR test that could be implemented in clinical settings could reduce women's risk of unplanned and mistimed pregnancies.

For research, HIV, STI, and pregnancy prevention interventions can evaluate participants for semen exposure biomarkers to address unintentional under-reporting of semen exposure including birth control sabotage, as well as intentional under-reporting, as has been recommended previously (Gallo et al. 2013; Snead et al. 2014). HIV, STI, and pregnancy prevention programs that include broad measures of educational attainment and employment can assess whether these human capital factors moderate the associations between coercion/sabotage and unprotected sex.

Acknowledgements The authors are grateful to Rachel Radigan for presenting this paper at the ICHPS 2020 conference, and for participants at presentations at the American Public Health Association conference in 2019 and at the research seminar at the SUNY Downstate School of Public Health.

Authors' contribution JR conceived the paper idea, analyzed the data, and wrote the manuscript. RD obtained funding, conceived the RCT study, and collected and cleaned the data. Both authors approved the final manuscript.

Funding This research was funded by the School of Public Health at SUNY Downstate Health Sciences University in Brooklyn, NY. The data collection was funded by 5R01AA018096 (Dr. DiClemente).

Availability of data and material The data have been submitted to ICPSR's National Addiction and HIV Data Archive Program https://www.icpsr.umich.edu/icpsrweb/NAHDAP/.

Code availability The Stata and R code is available as supplementary material. 


\section{Compliance with ethical standards}

Conflict of interest The authors declare that they have no conflict of interest.

Ethics approval Emory University's Institutional Review Board approved all study protocols (IRB00048502).

Consent to participate Informed consent was obtained from all individual participants included in the study.

Consent for publication Not applicable. This paper contains no individual identifiable information.

\section{References}

ACOG: ACOG Committee on Health Care for Underserved Women. ACOG Committee opinion no. 554: Reproductive and sexual coercion. Obstet. Gynecol. 121, 411-415 (2013)

Ali, M.S., Groenwold, R.H.H., Belitser, S.V., et al.: Reporting of covariates election and balance assessment in propensity score analysis is suboptimal: a systematic review. J. Clin. Epidemiol. 68, 112-121 (2015)

Austin, P.C.: A critical appraisal of propensity-score matching in the medical literature between 1996 and 2003. Stat. Med. 27, 2037-2049 (2008)

Belfield, C.R., Bailey, T.: The benefits of attending community college: a review of the evidence. Commun. Coll. Rev. 39(1), 46-68 (2011)

Brodsky A.: Rape-Adjacent': imagining legal responses to nonconsensual condom removal. Columbia J. Gender Law. 32 (2017)

Collins, L.M., Graham, J.W., Hansen, W.B., Johnson, C.A.: Agreement between retrospective accounts of substance use and earlier reported substance use. Appl. Psychol. Meas. 9, 301-309 (1985)

Connell, R.: Gender and Power: Society, the Person, and Sexual Politics. Stanford University Press, Standford (1987)

Copeland, K.T., Checkoway, H., McMichael, A.J., Holbrook, R.H.: Bias due to misclassification in the estimation of relative risk. Am. J. Epidemiol. 105(5), 488-495 (1977)

Crowder, K.D., Tolnay, S.E.: A new marriage squeeze for black women: the role of racial intermarriage by black men. J. Marr. Family 62, 792-807 (2000)

Gallo, M.F., Steiner, M.J., Hobbs, M.M., Warner, L., Jamieson, D.J., Macaluso, M.: Biological markers of sexual activity: tools for improving measurement in HIV/sexually transmitted infection prevention research. Sex. Transm. Dis. 40, 447-452 (2013)

Gelman, A., Hill, J.: Data Analysis using regression and multilevel/hierarchical models. Cambridge University Press, New York (2007)

Guttmacher Institute, Policy Brief: State Bans on Abortion Throughout Pregnancy, March 1, (2020). Retrieved from https://www.guttmacher.org/state-policy/explore/state-policies-later-abortions

Haine, D., Dohoo, I., Dufour, S.: Selection and misclassification biases in longitudinal studies. Front. Vet. Sci. 5, 99 (2018)

Hathaway, J.E., Willis, G., Zimmer, B., Silverman, J.G.: Impact of partner abuse on women's reproductive lives. J. Am. Med. Women's Assoc. 60, 42-45 (2005)

Ho, D., Imai, K., King, G., Stuart, E.A.: MatchIt: nonparametric preprocessing for parametric causal inference, software version 2.4-2. Harvard Institute for Quantitative Social Sciences (2018)

Höfler, M.: The effect of misclassification on the estimation of association: a review. Int. J. Methods Psychiatr. Res. 14(2), 92-101 (2005)

Imai, K., Keele, L., Tingley, D., Yamamoto, T.: “Causal mediation analysis using R”, Advances in Social Science Research Using R. Springer Lecture Notes in Statistics, New York, 129-54 (2010)

Klein, H.: Generationing, stealthing, and gift giving: the intentional transmission of HIV by HIV-positive men to their HIV-negative sex partners health. Psychol. Res.. 2, 1582 (2014)

Kolenikov, S., Angeles, G.: The use of discrete data in principal component analysis with applications to socio-economic indices tech. Rep. CPC/MEASURE Working paper No. WP-04-85 2004 (2004)

Laird, J.: Public sector employment inequality in the United States and the great recession. Demography 54, 391-411 (2017)

Liu, W., Kuramoto, S.J., Stuart, E.A.: An introduction to sensitivity analysis for unobserved confounding in non-experimental prevention research. Prev. Sci. 14(6), 570-580 (2013)

McCauley, H.L., Silverman, J.G., Jones, K.A., et al.: Psychometric properties and refinement of the reproductive coercion scale. Contraception 95, 292-298 (2017) 
Miller, E., Decker, M.R., McCauley, H.L., et al.: Pregnancy coercion, intimate partner violence and unintended pregnancy. Contraception 81, 316-322 (2010)

Miller, E., Decker, M.R., McCauley, H.L., et al.: A family planning clinic partner violence intervention to reduce risk associated with reproductive coercion. Contraception 83, 274-280 (2011)

Miller, E., Tancredi, D.J., Decker, M.R., et al.: A family planning clinic-based intervention to address reproductive coercion: a cluster randomized controlled trial. Contraception 94, 58-67 (2016)

Pallitto, C., Campbell, J.C., O'Campo, P.: Is intimate partner violence associated with unintended pregnancy? A review of the literature. Trauma Violence Abuse. 6, 217-235 (2005)

Paterno, M.T., Jordan, E.T.: A review of factors associated with unprotected sex among adult women in the United States. J. Obstet. Gynecol. Neonatal. Nurs. 41, 258-274 (2012)

Pettit, B., Ewert, S.: Employment gains and wage declines: the erosion of Black women's relative wages since 1980. Demography 46(3), 469-492 (2009)

Pouget, E.R., Kershaw, T.S., Niccolai, L.M., Ickovics, J.R., Blankenship, K.M.: Associations of sex ratios and male incarceration rates with multiple opposite-sex partners: potential social determinants of HIV/ STI transmission. Public Health Rep. 125, 70-80 (2010)

Rosenbaum, J.E., Zenilman, J.M., Rose, E., Wingood, G., DiClemente, R.J.: Cash, cars, and condoms: economic factors in disadvantaged adolescent women's condom use. J. Adolescent Health. 2012(51), 233-241 (2012)

Rosenbaum, J.E.: Degrees of health disparities: health status disparities between young adults with high school diplomas, sub-baccalaureate degrees, and baccalaureate degrees. Health Serv. Outcomes Res. Method. 12(2), 156-168 (2012)

Rosenbaum, J.E., Rosenbaum, J.E.: Money isn't everything: job satisfaction, nonmonetary job rewards, and sub-baccalaureate credentials. Res. High. Educ. J. 30, 1-17 (2016)

Rosenbaum, J.E., Zenilman, J.M., Melendez, J., Rose, E., Wingood, G.M., DiClemente, R.J.: Telling truth from Ys: an evaluation of whether the accuracy of self-reported semen exposure assessed by a semen Y-chromosome biomarker predicts pregnancy in a longitudinal cohort study of pregnancy. Sex. Trans. Infect. 90, 479-484 (2014a)

Rosenbaum, J.E., Zenilman, J.M., Rose, E., Wingood, G.M., DiClemente, R.J.: Do Jobs Work? Risk and protective behaviors associated with employment among disadvantaged female teens in urban Atlanta. J. Women Polit. Policy 35, 155-173 (2014b)

Rosenbaum, J.E., Zenilman, J.M., Rose, E., Wingood, G., DiClemente, R.J.: Predicting unprotected sex and unplanned pregnancy among urban african-american adolescent girls using the theory of gender and power. J. Urban Health. 93(3), 493-510 (2016)

Rosenbaum, J.E., Zenilman, J.M., Rose, E., Wingood, G.M., DiClemente, R.J.: Semen says: assessing the accuracy of adolescents' self-reported sexual abstinence using a semen Y-chromosome biomarker. Sex. Trans. Infections. (2016b)

Rosenbaum, J.E.: Reborn a virgin: adolescents' retracting of virginity pledges and sexual histories. Am. J. Public Health 96, 1098-1103 (2006)

Rosenbaum, J.E.: Truth or consequences: the intertemporal consistency of adolescent self-report on the youth risk behavior survey. Am. J. Epidemiol. 169(11), 1388-1397 (2009)

Rosenbaum, J.E.: Educational and criminal justice outcomes 12 years after school suspension. Youth Soc. 52(4), 515-547 (2020)

Rosenbaum, P.R.: Sensitivity analysis for m-estimates, tests, and confidence intervals in matched observational studies. Biometrics 63, 456-464 (2007)

Rowlands, S., Walker, S.: Reproductive control by others: means, perpetrators and effects. BMJ Sex. Reprod. Health 45, 61-67 (2019)

Silverman, J.G., McCauley, H.L., Decker, M.R., Miller, E., Reed, E., Raj, A.: Coercive forms of sexual risk and associated violence perpetrated by male partners of female adolescents. Perspect. Sex. Reprod. Health. 43, 60-65 (2011)

Snead, M.C., Black, C.M., Kourtis, A.P.: The use of biomarkers of semen exposure in sexual and reproductive health studies. J. Women's Health 23, 787-791 (2014)

Teitelman, A.M., Tennille, J., Bohinski, J.M., Jemmott, L.S., Jemmott, J.B.: Unwanted unprotected sex: condom coercion by male partners and self-silencing of condom negotiation among adolescent girls. Adv. Nurs. Sci. 34, 243-259 (2011)

Tingley, D., Yamamoto, T., Hirose, K., Keele, L., Imai, K. Mediation: R Package for causal mediation analysis. J. Stat. Softw. 59 (2014)

Tourangeau, R., McNeeley, M.E.: Measurement Problems in Criminal Justice Research. Measuring Crime and Crime Victimization: Methodological Issues. National Academy Press, Washington, DC (2003)

Trister, G.K., Anderson, J.C.: Reproductive coercion: a systematic review. Trauma Viol. Abuse. 19, 371390 (2018) 
Warnes, G.R., Bolker, B., et al.: gplots: Various R programming tools for plotting data, Library for the R statistical package (2019). Retrieved from https://cran.r-project.org/web/packages/gplots/index.html

Wingood, G.M., DiClemente, R.J.: Application of the theory of gender and power to examine HIV-related exposures, risk factors, and effective interventions for women. Health Educ. Behav. 27, 539-565 (2000)

Zenilman, J.M., Yuenger, J., Galai, N., Turner, C.F., Rogers, S.M.: Polymerase chain reaction detection of y chromosome sequences in vaginal fluid: preliminary studies of a potential biomarker for sexual behavior. Sex. Transm. Dis. 32, 90-94 (2005)

Publisher's Note Springer Nature remains neutral with regard to jurisdictional claims in published maps and institutional affiliations.

\section{Affiliations}

\section{Janet E. Rosenbaum ${ }^{1,2}$. Ralph J. DiClemente ${ }^{1,2}$}

1 Department of Epidemiology and Biostatistics, School of Public Health, SUNY Downstate Health Sciences University, Brooklyn, NY 11203, USA

2 Department of Social and Behavioral Sciences, College of Public Health, New York University, New York, NY, USA 